Introduction Recognising and harnessing cultural and technological shifts in both client and healthcare provider arenas are crucial to optimising clinic wide responses to STI education and care. As methods of client-to-service engagement evolve, healthcare providers and their documentation systems must change as well. This is crucial to optimising clinical care and interaction for potential sexual health clients, especially when incorporating mandatory area health service and ministry directives into daily practice.

Northern Sydney Sexual Health Service (NSSHS) triage has long allowed for telephone and walk-in enquiries. NSSHS has modified the existing service website to allow for online booking enquiries. This allows a potential patient to perform basic selftriage while engaging with sexual health services in a safe, nonconfronting manner. At the same time, this reduces telephone wait times for clients and improves engagement.

Results $75 \%$ of all website enquiries are appropriate for Clinic 16 services.

A significant increase in appointments made via our website enquiry system for priority populations has occurred since initiation in November 2014, including with men who have sex with men (MSM) and youth.

By improving our priority population engagement, there is a marked improvement in Occasions of Services and a projected improvement in Activity Based Funding (ABF) capture via the new electronic medical record system, Community Health and Outpatient Care system (CHOC).

Conclusion By integrating a multi-platform approach to patient engagement, NSSHS is growing to fit client needs while incorporating Local Health District and Ministry of Health mandated electronic medical record changes. This improves ABF capture, patient documentation and above all, improves engagement of priority populations with sexual health services.

Disclosure statement Northern Sydney Sexual Health Service is funded by the Northern Sydney Local Health District. No grants were received in the development of this study or project.

\section{P02.03 "CLICK YES TO CONSENT": CAN WE ACHIEVE INFORMED CONSENT FOR ONLINE TESTING FOR SEXUALLY TRANSMITTED INFECTIONS?}

${ }^{1} \mathrm{~A}$ Bonnell, ${ }^{2} \mathrm{D}$ Haag, ${ }^{2} \mathrm{M}$ Bondyra, ${ }^{2} \mathrm{~J}$ Farrell, ${ }^{2} \mathrm{M}$ Achen, ${ }^{3} \mathrm{E}$ Elliot, ${ }^{4} \mathrm{D}$ Unger, ${ }^{2,5,6} \mathrm{M}$ Gilbert ${ }^{*}$. ${ }^{1}$ Provincial Health Services Authority; ${ }^{2} B C$ Centre for Disease Control; ${ }^{3}$ College of Registered Nurses of British Columbia; ${ }^{4}$ Providence Health Care; ${ }^{5}$ University of British Columbia; ${ }^{6}$ Ontario HIV Treatment Network

\subsection{6/sextrans-2015-052270.224}

Introduction Achieving informed consent is integral to clinical testing, and imperative to consider when designing online testing services. We incorporated pre-test concepts necessary for informed consent into a new online STI/HIV testing service in British Columbia (GetCheckedOnline.com, GCO) and assessed the attitudes and understanding of this process.

Methods English-speaking participants $\geq 19$ years were recruited from Craigslist and among STI clinic clients for usability testing of a beta version of GCO, which included a webpage presenting 8 consent-related statements for review prior to printing a laboratory requisition. Participants were interviewed regarding their acceptability, perceptions, and understanding of the informed consent page; transcripts were analysed thematically.

Results We conducted 14 interviews (8 males, 6 females; 11 selfidentified as heterosexual; 8 previously tested at the provincial STI clinic). The consent page was acceptable to all and not perceived as a barrier or deterrent; many reported expecting to see a consent step in the process. While some viewed it as a formality or to reduce organisational liability, many participants were able to appropriately articulate the meaning and purpose of informed consent - perceiving it to be important for both the tester and the organisation, which for some led to increased trust and credibility of the service. Participants expressed the most concern regarding statements describing potential harms of testing and disease reporting. Compared to participants recruited through the STI clinic who related the step to their prior experience, participants with less testing experience generally demonstrated poorer understanding and awareness of concepts necessary for informed consent.

Conclusion We argue that principles of informed consent apply equally in online testing programs as in provider-led interactions, and can be effectively achieved without detracting from the user experience. As online testing models evolve, understanding how better to meet the informed consent needs of less-experienced testers is critical.

Disclosure of interest statement The authors have no conflicts of interest to disclose.

\section{P02.04 ACCEPTABILITY OF ONLINE RESOURCES FOR STI PARTNER NOTIFICATION: WHO WOULD USE WHAT IN THE TOOLKIT?}

1,2,3 M Gilbert*, 'S Wong, 'TS Hottes, ${ }^{1} \mathrm{D}$ Haag, ${ }^{1} \mathrm{~B}$ Brownrigg, ${ }^{1,2} \mathrm{~J}$ Wong, ${ }^{1,2} \mathrm{G}$ Ogilvie. ${ }^{1} B C$ Centre for Disease Control; ${ }^{2}$ University of British Columbia; ${ }^{3}$ Ontario HIV Treatment Network

\subsection{6/sextrans-2015-052270.225}

Introduction A variety of online STI partner notification (PN) resources have been developed to support people diagnosed with STI who elect to notify partners themselves. We conducted a survey of clients at a large urban STI clinic to determine the acceptability of different online resources for partner notification (PN). Methods Our waiting room survey was conducted over 8 months in Vancouver, British Columbia. We measured intention to use each of three online PN resources with a 5-point likelihood scale (dichotomized for analysis as likely/unlikely): email/ text notification service (using online form, anonymous option); sample letter/email; tips/videos about how to talk to partners. We used multivariable logistical regression to detect significant $(\mathrm{p}<0.05)$ associations between each outcome and potential explanatory factors.

Results Of 1539 clients surveyed, 26\% (email/text), 26\% (sample letter), and $68 \%$ (tips/videos) were likely to use each proposed service. Clients with more partners were more likely to intend to use the email/text service and tips/videos. Clients comfortable talking to partners were more likely to use the email/text service. Those who were likely to disclose all sex partners to a nurse were more likely to use the sample letter and tips/videos. The largest association was observed between intention to use email/ text service and preference for a 'mixed' approach to PN (client tells some, nurse tells some; OR 5.24 [95\% CI 3.43, 8.00]). Neither age nor gender of sex partners was associated with any of the outcomes.

Conclusion Results from this large survey suggest that acceptability of online PN resources is high, and may effectively supplement existing approaches to PN for some clients - notably those with multiple partners, and those preferring STI nurses to notify only some of these partners on their behalf. Having a variety of 
resources in an online PN "toolkit" available to support people diagnosed with STI is ideal.

Disclosure of interest statement The authors have no conflicts of interest to disclose.

\section{P02.05 SEXUAL HEALTH LITERACY - AN EMERGING FRAMEWORK FOR RESEARCH AND INTERVENTION TO IMPROVE SEXUAL HEALTH FOR GAY MEN}

${ }^{1,2,3} \mathrm{M}$ Gilbert ${ }^{*},{ }^{4,5} \mathrm{O}$ Ferlatte, ${ }^{3} \mathrm{~W}$ Michelow, ${ }^{6} \mathrm{~S}$ Martin, ${ }^{6}$ Y Young, ${ }^{7} \mathrm{~L}$ Donelle, ${ }^{8} \mid$ Rootman, ${ }^{6} \mathrm{~L}$ McDaid, ${ }^{9} \mathrm{P}$ Flowers. ${ }^{1} B C$ Centre for Disease Control; ${ }^{2}$ Ontario HIV Treatment Network; ${ }^{3}$ University of British Columbia; ${ }^{4}$ Simon Fraser University; ${ }^{5}$ Community-Based Research Centre for Gay Men's Health; ${ }^{6}$ University of Glasgow; ${ }^{7}$ Western University; ${ }^{8}$ University of Victoria; ${ }^{9}$ Glasgow Caledonian University

10.1136/sextrans-2015-052270.226

Introduction Health literacy is an important determinant of health yet has not been formally applied to sexual health. An expert event brought 38 researchers, community members, and service providers together to explore gay men's sexual health literacy (SHL).

Methods Using a World Café method, three rounds of discussion posed questions about SHL in relation to gay men, providers, and underlying systems. Documented notes were analysed thematically through two rounds of group synthesis and a subsequent review by one investigator.

Results SHL was influenced by ways men access information, through peer networks, and coded communications with prospective partners. The Internet influences access to, delivery of and engagement with information, while new technologies and changing sexual norms complicate message consistency and risk assessment. Actionable risk assessment requires numeracy skills. Gay men were generally perceived to have high SHL with concern that SHL may be lower for some sub-populations (e.g., youth, immigrant men). Participants valued bottom-up/community-based over top-down/expert models of providing SHL. Health care provider attitudes, cultural competency, knowledge and communication skills were seen as key determinants of SHL and non-traditional health sectors also play key roles (e.g., education, pornography). Underlying determinants of SHL included stigma related to HIV or sexual orientation, access and organisation of health care services, systemic shifts to self-care models, political ideologies and funding. Syndemic, intersectional, and holistic health approaches to SHL were advocated. Participants recognised the strong history of activism and mobilisation within gay communities as integral to SHL.

Conclusion Critical health literacy has been a core component of the gay men's health movement to date. Innovations in biomedical technologies and access to online information are critically shaping experiences of SHL for gay men. The expanded theoretical framework emerging from these findings serves as a starting point to inform enhancing SHL and designing effective interventions.

Disclosure of interest statement The authors have no conflict of interest to disclose.

\section{P02.06 IDENTITIES IN MOTION: CYBERSPACE AND MYANMAR MEN HAVING SEX WITH MEN}

Lin Khine Soe*, Putten Marc Van der*. Faculty of Public Health, Thammasat University, Rangsit Campus, Klong Luang Piyachart Bld. 10th Fl. Pathumthani, 12121, Thailand

10.1136/sextrans-2015-052270.227
The enquiry was financed by the World Society Foundations and the Thabyay Education Network.

This paper explores how Myanmar men-having-sex-with-men (MSM) create identity through lived and mediated realities, by using cyber social networks, 30 in-depth interviews were conducted through snowballing among MSM cyber social network members. Content analysis was guided by a social network analysis framework. Networking positively affected connectedness and social support however norms and values were challenged. Age, experience, sex-roles, and peer-relationships seem to influence self-efficacy. Interpersonal dynamics were driven by onlinecruising, dating, cyber-sex and off-line encounters. In conclusion, access to social networking offers an outlet for socio-cultural and legal oppressed Myanmar MSM, however building life-skills among younger users' needs attention. Social networks could be appropriate channels for social and health programs targeting MSM.

\section{P02.07 UTILISATION OF RISK SCORE TOOL OF INTERNET I WANT THE KIT (IWTK) HOME SELF-COLLECTION PROGRAM FOR SEXUALLY TRANSMITTED INFECTIONS (STIS) IN MALES}

${ }^{1} \mathrm{Y}$-H Hsieh*, ${ }^{2} \mathrm{M}$ Jett-Goheen, ${ }^{2} \mathrm{~L}$ Dize, ${ }^{2} \mathrm{M}$ Barnes, ${ }^{2} \mathrm{P}$ Barnes, ${ }^{1,2} \mathrm{CA}$ Gaydos. ${ }^{1}$ Department of Emergency Medicine; 'Infectious Diseases, Johns Hopkins University, Baltimore, Maryland, USA

\subsection{6/sextrans-2015-052270.228}

Introduction In our previous pilot study on the internet I Want The Kit (IWTK) home self-collection program for sexually transmitted infections (STIs), a voluntary risk score tool predicted STIs well for the female volunteers but not for the males. We investigated the association of IWTK risk score and the presence of STIs in male users when the risk score quiz became mandatory for the program.

Methods A six-question quiz which includes demographic and sexual risk behaviour became a mandatory part of IWTK in August 2103. This analysis was restricted to male participants living in Maryland and Washington DC using IWTK August 2013-April 2015. Cochran-Armitage trend test was performed to determine if the prevalence of STIs (chlamydia, gonorrhoea, or trichomonas infection) increased with the higher score of risk score category.

Results Overall, 592 male participants submitted specimens for STI testing and completed risk score quizzes. The majority $(57 \%)$ were $<30$ years (mean: $30.1 \pm 9.3$ years); $42 \%$ white, $42 \%$ black, and $16 \%$ other races. $203(34.3 \%)$ resided in zip codes of Baltimore City. The majority $(53 \%)$ of the participants had risk score of 4-6, followed by scores of $0-3(25 \%)$, and 7-10 (22\%). The overall prevalence of STIs was 10.5\% (62/ 592). The prevalence of STIs was $6.2 \%$ for users with risk score of $0-3,10.9 \%$ for those with $4-6$, and $14.3 \%$ for those with 7-10 (trend test: $p=0.026)$. 52\% (13/25) of STI positive participants resided in three zip codes located in central and northeast Baltimore City and participants in these three zip codes had a marginally higher risk score than others $(p=0.082)$.

Conclusions After excluding potential selection bias, IWTK risk score tool predicted the presence of STIs for male users and could potentially be used for identifying hotspots for STI intervention.

Disclosure of interest statement The research group has received research funding from U54EB007958, NIBIB, NIH; AI06861301, NIH, NIAID. 